Bull. Austral. Math. Soc.

VOL. 49 (1994) [117-119]

\title{
ON DISTINGUISHING SPACES NOT HOMOTOPY-EQUIVALENT
}

\author{
M.H. EgGaR
}

\begin{abstract}
A non-pathological example is given of two topological spaces which have isomorphic homotopy groups, homology groups and cohomology ring and which cannot be distinguished from each other by the Whitehead product structure. A family of examples can be constructed likewise.
\end{abstract}

Homology groups, the cohomology ring and homotopy groups are usually the first invariants that one meets in algebraic topology. Since for most spaces at least one of these is difficult to determine (indeed all three are rarely known explicitly), a very natural question is whether these invariants together suffice to distinguish between any two spaces that are not homotopy-equivalent. The spaces $S^{5} \times S^{4}$ and the Stiefel manifold of unit tangents to $S^{\mathbf{5}}$ provide the standard example of two spaces that have isomorphic homology groups, cohomology ring and homotopy groups. These spaces can however be distinguished by the Whitehead product structure on the homotopy groups $[3]$.

The purpose of this note is to present an example of two (non-pathological) spaces, namely $C P^{\infty} \times S^{3}$ and $\left(C P \times S^{1}\right) /\left(p t \times S^{1}\right)$, which have isomorphic homology groups, cohomology ring, homotopy groups and Whitehead product structure, but which are not homotopy-equivalent. The loop spaces of the two spaces are homotopy-equivalent. A family of examples can be constructed likewise. It should be remarked that in [2] Brayton Gray produced an example of two spaces that cannot be distinguished by an invariant carried by a finite skeleton and yet are not homotopy equivalent. His interesting construction exploits limiting operations to form a phantom map and then takes the reduced product of the mapping cone. Our example is not as powerful, but has the advantage of using only commonly occurring spaces and maps.

Let $\pi: S^{1} \times C P^{\infty} \rightarrow C P^{\infty}$ denote projection to the second factor, and let $E=$ $S^{1} \times C P^{\infty} \cup C S^{1}$ denote the mapping cone of the inclusion $S^{1} \rightarrow S^{1} \times C P^{\infty}$ of one fibre. Define $p: E \rightarrow C P^{\infty}$ to be the extension of $\pi$ that is constant on $C S^{1}$. The homotopy fibre of $p$ is homotopy-equivalent to $S^{3}$ by [1]. The map $p$ has a section $\sigma: C P^{\infty} \rightarrow E$ defined by $\sigma(b)=(1, b)$, hence $\sigma_{\#}+i_{\#}: \pi_{n} C P^{\infty} \oplus \pi_{n} S^{3} \rightarrow \pi_{n} E$ is an isomorphism for

Received 22 February 1993

Copyright Clearance Centre, Inc. Serial-fee code: 0004-9729/94 \$A2.00+0.00. 
each positive integer $n$, where $i$ is the inclusion of the homotopy fibre of $p$ into $E$ (up to the natural homotopy equivalences). The Puppe sequence $S^{1} \rightarrow S^{1} \times C P^{\infty} \rightarrow E$ yields $H^{n} E \simeq \mathbb{Z}$ for $n \geqslant 2$. (Hence the $E_{2}$-term of the cohomology spectral sequence for $p$, or strictly speaking for the map obtained when $p$ is converted to a fibration by the mapping path fibration construction [4], has zero differentials.)

The Whitehead products on $\pi_{*} E$ all vanish. For $u, v \in \pi_{*} S^{3}\left[i_{\#}(u), i_{\#}(v)\right]=$ $i_{\#}[u, v]=0$ by naturality of the Whitehead product and since $S^{3}$ is an $H$-space. Let $\omega$ be a generator of $\pi_{2} C P^{\infty}$. We now show $\left[\sigma_{\#}(\omega), i_{\#}(u)\right]=0$. Let $f: S^{2} \rightarrow$ $C P^{\infty}$ denote the inclusion $C P^{1} \subset C P^{\infty}$. Naturality of the mapping path fibration construction and Ganea's result gives a homotopy commutative diagram

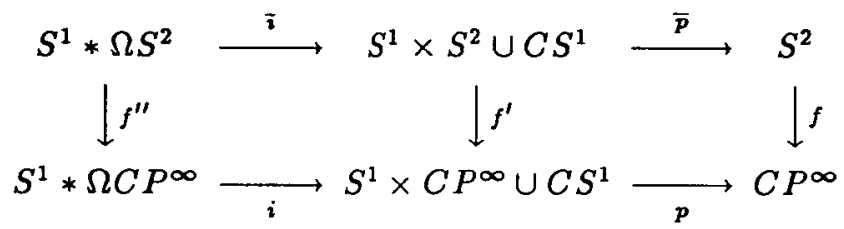

where $f^{\prime \prime}=1_{S^{1}} * \Omega f, f^{\prime}\left|S^{1} \times S^{2}=1_{S^{1}} \times f, f^{\prime}\right| C S^{1}=1_{C S^{1}}$ and $\bar{\imath}, \bar{p}$ are defined in the analogous way to $i, p$. Let $\bar{\sigma}: S^{2} \rightarrow S^{1} \times S^{2} \cup C S^{1}$ be the section of $\bar{p}$ defined by $\bar{\sigma}(b)=(1, b)$. We note that $S^{1} * \Omega S^{2}$ is homotopy-equivalent to $S^{3} \vee S^{4} \vee S^{5} \vee \cdots$, $S^{1} \times S^{2} \cup C S^{1}$ is homotopy-equivalent to $S^{2} S^{3}, f^{\prime \prime} \mid S^{3}=1_{S^{3}}$ and $f^{\prime \prime} \mid S^{4}{ } S^{5} \vee \ldots$ is null homotopic. Let $\bar{\omega}$ denote the generator of $\pi_{2} S^{2}$ such that $f_{\#}(\bar{\omega})=\omega$ and let $j$ : $S^{3} \rightarrow S^{3} \vee S^{4} \vee S^{5} \vee \ldots$ denote the inclusion onto the first summand in the wedge. Then $f_{\#}^{\prime \prime} j_{\#}(u)=u$. When $\pi_{*}\left(S^{2} \vee S^{3}\right)$ is expanded as $\bar{\sigma}_{\#} \pi_{*} S^{2} \oplus \bar{\imath}_{\#} j_{\#} \pi_{*} S^{3} \oplus \sum_{n \geqslant 4} \bar{\imath}_{\#} \pi_{*} S^{n}$, by the Hilton Milnor theorem $\left[\bar{\imath}_{\#} j_{\#}(u), \bar{\sigma}_{\#}(\bar{\omega})\right]$ lies in the latter summands. Since $f^{\prime \prime} \mid S^{4} \vee S^{5} \vee \ldots$ is null homotopic, $f_{\#}^{\prime}\left[\bar{\imath}_{\#} j_{\#}(u), \bar{\sigma}_{\#}(\bar{\omega})\right]=0$. Hence

$$
\left[\sigma_{\#}(\omega), i_{\#}(u)\right]=\left[\sigma_{\#} f_{\#}(\bar{\omega}), i_{\#} f_{\#}^{\prime \prime} j_{\#}(u)\right]=\left[f_{\#}^{\prime} \bar{\sigma}_{\#}(\bar{\omega}), f_{\#}^{\prime} \bar{z}_{\#} j_{\#}(u)\right]=0
$$

It is straightforward to show that $S^{3} \times C P^{\infty}$ has homotopy groups, Whitehead product structure, homology groups and cohomology ring isomorphic to those for $E$. It remains to show that $E$ is not homotopy-equivalent to $S^{3} \times C P^{\infty}$. This is easily done by calculating the Steenrod square $\mathrm{Sq}^{2}$ on $H^{3}\left(-; Z_{2}\right)$. Alternatively one may proceed as follows. Suppose there were a homotopy equivalence $g=\left(g_{1}, g_{2}\right): E \rightarrow S^{3} \times C P^{\infty}$. Then $g_{1}^{*}: H^{3}\left(S^{3}\right) \rightarrow H^{3}(E)$ would be an isomorphism. Let $q: S^{n} \times S^{m} \rightarrow S^{n+m}$ denote the quotient map that identifies $S^{n}{ } S^{m}$ to a point. Consider the composite map $h=g_{1} \circ e \circ\left(1_{S^{1}} \times f\right): S^{1} \times S^{2} \rightarrow S^{1} \times C P^{\infty} \rightarrow E \rightarrow S^{3}$, where $e$ denotes the inclusion of $S^{1} \times C P^{\infty}$ into $E$. Now $h^{*}: H^{3}\left(S^{3}\right) \rightarrow H^{3}\left(S^{1} \times S^{2}\right)$ is an isomorphism, since each of $g_{1}^{*},\left(1_{S^{1}} \times f\right)^{*}$ and $e^{*}$ is an isomorphism on $H^{3}$. Also $h \mid S^{1} \vee S^{2}$ is null homotopic, so $h$ factors through the quotient map $q: S^{1} \times S^{2} \rightarrow S^{3}$ to give a map $k: S^{3} \rightarrow S^{3}$ 
such that $h=k \circ q$. Since $k^{*}$ is an isomorphism, $k$ is a homotopy equivalence. Let $\eta: S^{3} \rightarrow S^{2}$ denote the Hopf map. The composite map $h \circ\left(1_{S^{1}} \times \eta\right): S^{1} \times S^{3} \rightarrow$ $S^{1} \times S^{2} \rightarrow S^{3}$ extends over $S^{1} \times D^{4}$, since $h$ extends over $S^{1} \times\left(4-\right.$ cell of $\left.C P^{\infty}\right)$, and hence $h \circ\left(1_{s^{1}} \times \eta\right)$ is null homotopic. On the other hand consider the commutative diagram

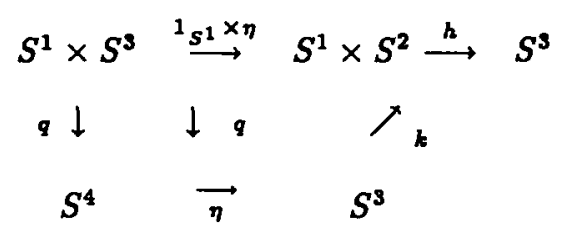

As $k$ is a homotopy equivalence $k \circ \eta: S^{4} \rightarrow S^{3}$ is not null homotopic, in contradiction to

Lemma. A map $f: S^{n+m} \rightarrow Y$ is null homotopic if $f \circ q: S^{n} \times S^{m} \rightarrow Y$ is null homotopic.

Proof: The Puppe sequence $S^{n} v S^{m} \underset{t}{\longrightarrow} S^{n} \times S^{m} \underset{q}{\longrightarrow} S^{n+m}$ yields the exact sequence

$\left[S^{n}{ } S^{m}, Y\right] \longleftarrow\left[S^{n} \times S^{m}, Y\right] \underset{q^{\#}}{\longleftarrow}\left[S^{n+m}, Y\right] \longleftarrow\left[S\left(S^{n} \vee S^{m}\right), Y\right] \longleftarrow\left[S\left(S^{n} \times S^{m}\right), Y\right]$.

Since $\left[S\left(S^{n} \times S^{m}\right), Y\right]=\left[S^{n+1} \vee S^{m+1} \vee S^{n+m+1}, Y\right]$ we have $t^{\#}$ is onto, hence $\partial=0$, hence $q^{\#}$ is injective.

\section{REFERENCES}

[1] T. Ganea, 'A generalization of the homology and homotopy suspension', Comm. Math. Helv. 39 (1964), 295-322.

[2] B.I. Gray, 'Spaces of the same n-type, for all n', Topology 5 (1966), 241-243.

[3] I.M. James and J.H.C. Whitehead, 'Homotopy theory of sphere bundles over spheres', Proc. London Math. Soc. 4 (1954), 196-218.

[4] E.H. Spanier, Algebraic topology (McGraw Hill, New York, 1969), pp. 99.

Department of Mathematics and Statistics

The University of Edinburgh

Edinburgh EH9 3JZ

Scotland 